\title{
Jornalismo de Saúde: Prevenir ou Remediar?
}

\author{
EMILIANA SOFIA GOMES \\ Universidade do Minho - Portugal - sofiagomes_11@hotmail.com \\ Mestre em Ciências da Comunicação na vertente Informação e Jornalismo. \\ Foi colaboradora do projeto "A Doença em Notícia" e é, atualmente, \\ bolseira de investigação no projeto "Estação NET", do Centro \\ de Estudos de Comunicação e Sociedade da Universidade do Minho.
}

\begin{abstract}
Resumo
Neste artigo trabalhou-se o tema da prevenção na imprensa portuguesa, procurando-se responder à seguinte questão de partida: "Até que ponto os artigos de saúde dos jornais Público, Jornal de Notícias e Expresso revelam um papel de prevenção por parte destes meios de comunicação?". O estudo revelou que poucos são os textos publicados com o intuito de prevenir a doença e promover a saúde, registando-se apenas 43 artigos preventivos num universo de 1890 textos publicados sobre saúde. O trabalho comprovou também a existência de um perfil estereotipado da fonte de informação em saúde. As questões da Comunicação em Saúde, Promoção da Saúde e Educação para a Saúde são os alicerces que permitiram estudar a prevenção no campo da saúde.
\end{abstract}

\section{Palavras-chave}

comunicação em saúde, prevenção, fontes de informação

\begin{abstract}
In this paper, we made an observation about the theme related with prevention in Portuguese press, in order to answer the following question: "To what extent health articles of newspapers, Jornal de Notícias and Expresso reveal a role of prevention of these media?". The study proved that are few published texts to prevent disease and promote health, registering only 43 preventive in a universe of 1890 published articles about health. The work also demonstrated the existence of a stereotypical profile of health information source. Issues of Health Communication, Health Promotion and Health Education are the foundation that allowed to study prevention in health field.
\end{abstract}

Keywords

Health Communication, prevention, sources of information

Artigo recebido em 10/09/2012

Aprovado em 26/09/2012 
$\int \begin{aligned} & \text { á anos que a temática da saúde vem sendo tratada como um campo frutífero } \\ & \text { nos estudos em comunicação. Contudo, em Portugal, os avanços realizados } \\ & \text { não acompanham o contexto internacional. }\end{aligned}$

\section{Saúde: um conceito em mutação}

Etimologicamente, saúde tem origem na expressão latina salus que, de acordo com Naomar Filho (2000), dizia respeito ao atributo principal dos inteiros, intactos, íntegros. Porém, a noção de saúde vive em permanente mutação. A Organização Mundial de Saúde (OMS), em 1985, descreveu o conceito enquanto a "capacidade para realizar o potencial pessoal e responder de forma positiva aos desafios do ambiente". Um ano mais tarde, na Carta de Otava, definiu saúde como um "recurso para a vida e não uma finalidade" (Direção Geral de Saúde (DGS), 2003). Mas as definições não cessam aqui. Thorese e Eagleston (1985) apontam o conceito de saúde como "um estado em que está subjacente a capacidade de a pessoa realizar as atividades de vida diárias"(in Araújo, 2004: 39). Já Carvalho e Carvalho lembram a definição de Déjours: "Saúde é a capacidade de cada homem, mulher ou criança criar e lutar pelo seu projeto de vida, pessoal e original, em direção ao bem-estar" (2006: 49). Esta conceção acentua o dinamismo do conceito de saúde, anunciando a "capacidade funcional do ser humano" e a "indicação de géneros e grupos etários" (Carvalho \& Carvalho, 2006: 7).

Mais realista, Xavier (2006: 50) salienta que "saúde é mercadoria", e que as "tentativas de apropriação do conceito obedecem a uma finalidade quase única: a comercialização". Vê-a como uma cura, "amparada em dicotomias do senso-comum e descontinuidades, definindo-se, assim, a partir de um «negativo» a suplantar" (Xavier, 2006: 51). Paralelamente, cria uma analogia entre saúde e tecnologia, resultando isto no que chama de "cientifização" da saúde. A importância dada à tecnologia enquanto sinónimo de eficácia, qualidade e progresso prova, assim, que vivemos num tempo "totalmente cientificista, no qual as novidades tecnológicas passam a ter estatuto de qualidade e eficácia somente pelo facto de serem novidades" (ibidem).

\section{A influência da comunicação na promoção da saúde}

Comunicação em Saúde ${ }^{i}$ começou a ser reconhecida como área de investigação entre 1970 e 1990. Atualmente torna-se difícil definir o conceito em virtude da infinidade de propostas. Contudo, todas seguem o mesmo pensamento: o objetivo de 
Estudos em Jornalismo e Mídia - Vol. 9 № 2 - Julho a Dezembro de 2012

compreender a influência da comunicação humana mediada na prestação e promoção de cuidados de saúde às populações.

Para Renaud e Sotelo, define-se como "o estudo e utilização de estratégias de comunicação interpessoais, organizacionais e mediáticas destinadas a informar e influir nas decisões individuais e coletivas propícias à melhoria da saúde" (2007: 215). De acordo com a OMS, citada por Terrón (s/d: 3), “abarca várias áreas que incluem a educação (...) a comunicação interpessoal, a advocacia para a saúde nos meios de comunicação, a comunicação dentro das organizações, a comunicação sobre riscos, a comunicação social, o marketing social (...)". A Comunicação em Saúde está, assim, presente em diversos contextos.

José Teixeira (2004: 615) define-a como o "estudo e utilização de estratégias de comunicação para informar e para influenciar as decisões dos indivíduos e das comunidades no sentido de promoverem a sua saúde" e Márcia Moisés (2003) considera-a "uma chave, dentro do processo educativo, para compartilhar conhecimentos e práticas que podem contribuir para a conquista de melhores condições de vida".

\section{Prevenção: uma missão da comunicação}

Ferreira (1986) explica que “o termo 'prevenir' tem o significado de «preparar; chegar antes de; dispor de maneira que evite (dano, mal); impedir que se realize»»" (Czeresnia, 2003: 4). Anos mais tarde, Moreira (2005:13) afirma que "prevenir significa desenvolver atividades que impeçam ou dificultem a chegada de determinada condição", e alerta para a necessidade de "conhecer os fatores precursores da mesma condição" de modo a saber-se utilizar as estratégias mais corretas. Para o autor, "a valorização da prevenção como estratégia foi acompanhada de evoluções no próprio conceito de prevenção" (Moreira, 2005:12). Resumidamente, "a prevenção em saúde «exige uma ação antecipada, baseada no conhecimento da história natural a fím de tornar improvável o progresso posterior da doença» (Leavell \& Clarck, 1976: 17)”, sendo que as ações preventivas são orientadas para evitar o surgimento de doenças específicas, reduzindo sua incidência e prevalência nas populações” (Ferreira, 1986).

Pacheco lembra que "mais importante que a atuação punitiva assegurada por Lei, cabe-lhes [aos media] a missão preventiva", ou seja, o autor acredita que "é por meio de informações com qualidade e ética que poderemos mudar as atitudes e comportamentos, bem como transformar a sociedade" (Pacheco, 2005: 9). 


\section{Educar para a Saúde}

Lawrence Green define Educação em Saúde como "qualquer combinação de oportunidades de aprendizagem projetada para facilitar a adaptação voluntária de comportamento que irá melhorar ou manter a saúde" (in Hubley, 1993: 17). A noção de uma adaptação voluntária é uma ideia a reter e com contornos éticos bastante importantes, pois “implica que os educadores de saúde não devem forçar as pessoas a fazer o que eles não querem fazer" (ibidem).

Também o significado de Educação para a Saúde tem vindo a sofrer alterações. Carvalho e Carvalho (2006: 20) recordam que, em 1926, Wood definia-a como uma soma de experiências e impressões que tinham influência direta nos hábitos, conhecimentos e atitudes dos indivíduos na saúde. Apesar das mudanças recentes, esta definição pioneira já abordava questões como as atitudes e os comportamentos ou ainda a dimensão dos conhecimentos. Porém, também preteria aspetos fundamentais do ponto de vista atual, como os valores e as crenças dos indivíduos ou as suas necessidades.

Para Carvalho e Carvalho, nenhuma destas definições incluem aspetos essenciais como a autoestima, a auto capacitação ou os valores individuais. Por isso, apontam a definição de Tones e Tilfors (1994) como uma das mais completas e bem aceites.

Educação para a saúde é toda a atividade intencional conducente a aprendizagens relacionadas com saúde e doença [...], produzindo mudanças no conhecimento e compreensão e nas formas de pensar. Pode influenciar ou clarificar valores, pode proporcionar mudanças de convicções e atitudes; pode facilitar a aquisição de competências; pode ainda conduzir a mudanças de comportamentos e de estilos de vida. (Tones \& Tilfors, 1994: 11)

De acordo com Lopes et al. (s/d), o conceito de literacia abrange "a capacidade de entender e usar a informação escrita nas atividades diárias de modo a atingir os objetivos pessoais e desenvolver o seu próprio conhecimento e potencial (Friedman \& Hoffman-Goetz, 2010: 286)". Para as autoras, "literacia em saúde afeta, particularmente, o nosso conhecimento acerca dos cuidados de saúde, a nossa capacidade de encontrar e comunicar informação sobre saúde e a nossa competência para tomar decisões críticas” (ibidem). Neste sentido, quer a Educação para a Saúde, quer a Literacia surgem como "ferramentas fundamentais para o processo de 
Estudos em Jornalismo e Mídia - Vol. 9 № 2 - Julho a Dezembro de 2012

capacitação (ou empowerment) das populações" (in Carvalho et al., 2008: 3), tendo um papel fundamental na prevenção de comportamentos de risco.

\section{Comunicação como motor de promoção da saúde}

De acordo com Renaud e Sotelo (2007: 216), a comunicação "constitui um potente motor de promoção da saúde", que é preferencialmente feita através da prevenção e, para Gary Kreps, é essencial para incentivar o público a detetar precocemente qualquer tipo de doença ou, por exemplo, para "promover o desenvolvimento de programas de rastreio na área da saúde" (2003: 15).

Nutbeam (2006: 261) salienta que "os resultados da promoção da saúde representam fatores pessoais, sociais e estruturais que podem ser modificados com o objetivo de alterar determinantes da saúde". A promoção implica, então, o "fortalecimento da capacidade individual e coletiva para lidar com a multiplicidade dos condicionantes de saúde", indo além da "aplicação técnica e normativa" (ibidem).

Desta forma, para Lopes et al. (s/d), "a promoção da saúde compreende o desenvolvimento e difusão de mensagens persuasivas dirigidas a públicos segmentados, com vista a influenciar perceções, atitudes e comportamentos".

Nos dias de hoje, as notícias de saúde são tidas como um produto cujo objetivo é ser vendido, sendo a saúde vista como uma mercadoria. Neste sentido, os meios de comunicação tendem a afastar-se dos verdadeiros problemas que inquietam a população, sendo a participação dos media na promoção da saúde quase nula. Em algumas circunstâncias, os media conseguem até ser contra "certos pressupostos deste processo". Isto acontece, pois, tendencialmente, veem a saúde como a ausência da doença, apoiando o consumo de determinados produtos para curar as ditas doenças (Lefèvre, 1999). Por isso, para Bydlowski et al. (2004), a cooperação dos media seria uma mais-valia no processo de promoção de saúde, tendo em conta que podiam ajudar a valorizar o ser humano enquanto cidadão e facilitar relacionamentos menos opressivos na sociedade.

\section{Fontes de informação}

O estudo das fontes ${ }^{\mathrm{iii}}$ noticiosas é uma questão incontornável na investigação em jornalismo e a difusão de informação sobre saúde registou um aumento significativo 
por volta da década de 1990, marcando o início de uma tendência que se desenvolveu nos anos subsequentes.

Denise Gomes (2010: 6) refere que as fontes "são parte do processo de produção da notícia" e Pellegrini (2008: 279) acrescenta que "são um elemento primordial para qualquer tentativa de entendimento do discurso periodístico na atualidade", isto porque "não há jornalismo sem fonte" (ibidem). Walter Junior (2006: 118) realça que "as fontes são mais importantes para o processo de produção de notícias que o próprio jornalista, uma vez que são elas que oferecem a matéria-prima da notícia”. De uma forma mais completa, Mario Erbolato, citado por Lopez (2009: 2), estabelece que "a fonte é todo aquele sujeito que possa, de alguma maneira, transmitir informações sobre um dado acontecimento ao jornalista". Para Gomis (1991:59), "as fontes podem ser pessoas, grupos, instituições sociais, ou são ainda vestígios, discursos, documentos, dados, por aqueles deixados ou construídos", sendo que "remetem a posições e relações sociais, para interesses e pontos de vista, para quadros espaciais temporalmente situados" (ibidem). Assim, no campo específico da saúde, o objetivo de ter uma informação equilibrada parece ser um propósito difícil de concretizar, sendo a construção de relações consistentes com fontes acessíveis, credíveis e fiáveis um aspeto a considerar neste contexto.

\section{Opções metodológicas}

Este trabalho tem como base de investigação o projeto "A Doença em Notícia", pelo que a metodologia deriva, essencialmente, do projeto.

Em termos práticos, foram selecionadas todas as notícias de saúde - 1890 artigos -, criando-se uma base de dados que inclui informações sobre os artigos de saúde publicados em três jornais portugueses: 733 artigos no Público, 1038 no Jornal de Notícias (JN) e 119 no Expresso.

A amostragem pretendida resultou de um método de seleção não probabilístico e a justificação desta escolha começa por estar nos recursos disponíveis, na facilidade de acesso aos dados dos três jornais e nos interesses do projeto. Além disso, a opção por estes jornais (um semanário de referência - Expresso - e dois diários, um de referência e outro de cariz mais popular - Público e $J N$, respetivamente), compreende-se pelo facto de ser uma amostragem onde a periodicidade distinta aponta para a hipótese de que possivelmente existem diferenças no tratamento dado por jornais que tenham 
periodicidade diária ou semanal e, numa outra hipótese, que tenham uma linha editorial de referência ou mais popular.

Tendo por base a pergunta de partida: “Até que ponto os artigos de saúde dos jornais Público $^{i v}$, Jornal de Notícias ${ }^{v}$ e Expresso ${ }^{v i}$ revelam um papel de prevenção por parte destes meios de comunicação?” e com os artigos resultantes desta seleção, tentouse fazer uma análise de conteúdo que procurou responder a variáveis como: presença/ausência de referência às fontes ${ }^{\mathrm{vii}}$; número de fontes citadas; lugar geográfíco onde se situa a fonte; género; identificação; estatuto; especialidade médica; motivo de noticiabilidade. Tratando-se de um mapeamento extensivo, optou-se por uma análise quantitativa dos dados, através de uma estatística descritiva univariada, tornando-se possível fazer um levantamento de regularidades no corpus e posterior medição e associação de dados.

O período estudado varia entre os dias 1 de Janeiro e 31 de Dezembro de 2011.

\section{Mais vale prevenir que remediar}

Constata-se que o $J N$ é a publicação com maior número de artigos publicados sobre saúde, representando $54,9 \%$ do corpus. De seguida está o Público com um valor relativamente mais baixo - 38,8\% e, por fim, o Expresso com 6,3\%. Embora a disparidade de valores entre o $J N$ e o Público seja difícil de explicar, o número de artigos apresentado pelo Expresso compreende-se pelo facto de este ser um jornal semanário, o que, naturalmente lhe confere um número de textos publicados mais reduzido, seja qual for a temática em causa.

Para traçar o perfil dos textos publicados sobre saúde, é possível também considerar a variável "tipo de artigo", sendo legítimo afirmar-se que as "notícias" são o tipo de artigo privilegiado pelos jornalistas, registando-se 1684 textos em 1890, ou seja, uma percentagem correspondente a 89,1\%. Os restantes artigos distribuem-se em "reportagem" com 8,7\% e "entrevista" com 2,2\%. Esta situação justifica-se, talvez, com fatores como constrangimentos de tempo, dinheiro, número de profissionais, equipamento, transporte, espaço, entre outros como as próprias linhas editoriais. Verifica-se também que, comparadas com os restantes tipos de artigo, as reportagens abundam mais no jornal Expresso. Esta abundância de reportagens acontece, pois, dos três jornais em estudo, este é o único que tem uma publicação semanal (e não diária), o que permite dar ao jornalista algum tempo para trabalhos mais extensos e complexos, como são os casos das reportagens e das entrevistas. 
Os dados recolhidos permitiram, ainda, perceber que, num total de 1890 artigos, 849 deles apresentam títulos negativos $-44,9 \%$. Isto significa que, à partida, quase metade dos artigos terão uma abordagem menos positiva relativamente ao tema noticiado. Em segundo lugar ficaram os títulos neutros/ambíguos que representam 37\% e, por fim, os títulos positivos são deixados para o último lugar com apenas $18 \%$.

Um outro aspeto observado no corpus foi o "tempo da notícia". Aquilo que se pretende com esta variável é perceber a que período de tempo se refere o artigo publicado, ou seja, saber se o texto foi escrito antes, no dia ou depois do acontecimento noticiado. Posto isto, verifica-se uma primazia da opção "dia anterior", que apresenta $35,8 \%$, depois está a opção "ponto de situação" com 34,9\% e, já mais afastados, temos "não sei data" com 15\%, "antecipação de eventos" com 10,5\% e "mais de um dia" com $3,7 \%$. O que se pode constatar é que, em grande parte, os artigos retratam situações que aconteceram no dia anterior e isto verifica-se muito regularmente através da expressão “ontem". Já em relação ao "ponto de situação" acontece quando o artigo dá conta de uma atualização de alguma notícia já dada aos leitores.

Outra variável contabilizada foi o "tamanho" do artigo. Aqui, verifica-se que, na sua grande maioria, os artigos são médios, registando-se $54,2 \%$ dos textos publicados. Com valores mais baixos estão os textos extensos, com $29,2 \%$ e os breves com $16,6 \%$. Também aqui os resultados se explicam por uma série de constrangimentos, tais como as linhas editoriais de cada jornal; fatores económicos; tempo para recolha de informação e construção do texto; constrangimentos de espaço, como por exemplo, a imposição de limite de caracteres para a escrita do artigo, entre muitos outros fatores. $\mathrm{O}$ "tamanho do artigo" é também interessante para perceber o "valor" atribuído por um jornal a determinado assunto. À partida, quanto maior for a notícia, maior destaque será dado ao caso. Assim sendo, o facto de os artigos breves serem em menor número demonstra que os assuntos de saúde são valorizados pelos jornais.

Os resultados da variável "lugar da notícia" são, talvez, dos que mais surpreendem, se se tiver em linha de conta a conhecida primazia da zona de Lisboa, enquanto foco de densidade da concentração noticiosa nacional.

Verifica-se, então, que quase metade dos artigos publicados tem origem na área considerada "nacional global", registando-se 888 textos dos 1890 que compõem o corpus, ou seja, $47 \%$. Os restantes valores estão, porém, bastante dispersos, embora se destaque os casos do "Norte" e de "Lisboa e Vale do Tejo". No caso do "Norte", os valores são surpreendentes - 18,1\% - no entanto explicáveis se pensarmos que o $J N$ é a 
publicação que mais escreve em saúde e que é um jornal nortenho. Esta situação é, aliás, corroborada pelo cruzamento das variáveis "lugar da notícia" e "jornal". Já no caso de "Lisboa e Vale do Tejo" os valores apontam para os 12,8\%, o que se explica pela tendência dos jornalistas em dar atenção aos assuntos da capital, nomeadamente por questões como proximidade das redações, concentração de instituições públicas, entre outras.

Depois deste panorama geral, está-se agora em posição para refletir sobre outra variável: o "motivo de noticiabilidade", fazendo aqui uma referência direta à questão da prevenção, que é, como já se constatou, um dos pontos fulcrais deste estudo.

Os dados revelam que "prevenção" é um motivo deixado de parte pelos jornais, registando-se apenas 2,3\%, o que significa apenas 43 artigos num universo de 1890 . Estes resultados afiguram-se preocupantes, uma vez que o papel preventivo dos meios de comunicação social, em particular da imprensa portuguesa, está a ser desvalorizado e altamente subaproveitado.

Vistas as variáveis que dizem respeito ao perfil geral dos artigos, passa-se agora ao caso específico das fontes de informação.

Segundo os resultados obtidos, sabe-se, então, que 97,4\% dos artigos registam a presença de fontes de informação, contrapondo-se apenas $2,6 \%$ em que isto não acontece. Tendo em conta que um dos objetivos deste trabalho é precisamente estudar o caso das fontes, considera-se que os valores são estimulantes e positivos.

Destes $97,4 \%$ dos textos, sabe-se que 35,2\% deles apresentam apenas uma fonte, $26,6 \%$ duas fontes, $20,2 \%$ quatro ou mais e $15,4 \%$ três fontes de informação, assim como, em termos práticos, estes 1841 textos dão origem a 4510 fontes de informação.

Relativamente à sua geografia, os dados revelam que mais de metade das fontes é "Nacional", o que na prática se converte em 2430 casos dos 4512 possíveis, ou seja, 53,9\%. Com valores bastante mais reduzidos temos o "Norte", cuja percentagem equivale a 13,7\%, seguindo-se "Lisboa e Vale do Tejo" com 8,9\%. A explicação para estes dados está intrinsecamente relacionada com a tendência dos profissionais para privilegiarem as informações de âmbito nacional ou, por outro lado, provenientes da zona de Lisboa devido a fatores económicos, de tempo, de acessibilidade ou até mesmo de conveniência ou em função de determinadas linhas editoriais.

A análise das fontes de informação durante o ano 2011 permite-nos também fazer algumas observações relativamente ao "género" mais chamado a falar. Assim 
sendo, a fonte masculina é a preferida pelos jornalistas, registando-se 1988 casos num universo de 4512, ou seja, 44,1\%. Com valores mais reduzidos, segue-se a fonte "não pessoal", com $27,9 \%$ e só depois a fonte "feminina", com 15,6\%. Em último lugar ficam as fontes "coletivas" com 9,4\% e "não sei" com $3 \%$.

Uma outra variável que também parece importante de analisar é a “identificação das fontes”. Esta variável revela que há uma elevada percentagem de fontes identificadas - 85,9\% - correspondente a 3878 casos num total de 4512 . Relativamente a fontes "não identificadas" o valor é mais reduzido, ficando-se pelos $13,5 \%$ e as fontes "anónimas" registam apenas $0,6 \%$.

Segundo os dados, os valores recolhidos relativamente ao estatuto das fontes são muito dispersos, embora se destaque um maior número de fontes "especializadas institucionais - médicos no campo da saúde" e "media e sites noticiosos", ambos com 9,9\%. Um valor também bastante interessante é o referente aos "pacientes e familiares", que registam 5,7\%, ou seja, 257 das 4512 fontes estudadas. Embora não seja uma percentagem muito elevada, revela um interesse por parte dos jornalistas em relação ao cidadão comum, isto é, às fontes que não representam uma instituição ou um órgão oficial, o que, por si só, é um dado de grande relevância.

Relativamente ao estatuto da fonte, sobressai um outro aspeto: a existência de mais fontes dentro do campo da saúde - 2805 casos - do que fora do campo - 1623 fontes.

A base de dados permite também verificar a questão da "especialidade da fonte", ou seja, perceber qual a área da medicina em que trabalham as fontes contabilizadas como médicos na variável "estatuto". Assim, de um modo geral, as fontes médicas mais utilizadas são as incluídas na gaveta "oncologia”, com 10\%, depois "medicina geral e familiar", com 6,9\% e "cirurgia" com 6,3\%. Para o fim são deixadas especialidades como Anestesiologia, Endocrinologia e Nutrição, Gastrenterologia, Hematologia Clínica, Medicina Nuclear, Ortopedia e Radiodiagnóstico, todas com $0,6 \%$.

\section{Notas conclusivas}

Com este estudo procurou-se perceber se existe, ou não, um papel preventivo da imprensa portuguesa relativamente a casos de saúde, ou seja, responder à questão de partida: "Até que ponto os artigos de saúde dos jornais Público, Jornal de Notícias e 
Expresso revelam um papel de prevenção por parte destes meios de comunicação?". Contudo, responder a isto tornou-se um pouco complexo.

Com a observação dos dados, concluiu-se, pois, que atualmente, as notícias de saúde são encaradas pelos media como um produto cujo objetivo final é ser vendido. À semelhança do que acontece com as outras notícias, toda a área da saúde é vista como uma mercadoria, situação que não favorece o desenvolvimento de processos de promoção e prevenção da saúde. Quanto a este assunto apraz lembrar a questão da "medicalização" referida por McAllister (1992), assim como a forma como a Medicina altera a perceção que as pessoas fazem da realidade.

Em jeito de conclusão, parece igualmente importante refletir sobre o próprio conceito de Comunicação em Saúde, cujos objetivos, perante as definições encontradas e os resultados obtidos com o estudo, não são totalmente cumpridos na imprensa portuguesa.

A imprensa não parece compreender a influência que tem na prestação e promoção de cuidados de saúde à população. Contudo, conforme se verifica, confirmase que a Comunicação em Saúde funciona como resposta a interesses políticos. Isto é particularmente visível se se pensar na forte presença de fontes oficiais, ou seja, fontes ligadas ao Governo, dentro ou fora do campo da saúde.

Pensando no outro conceito aqui abordado - prevenção - pode também concluir-se que claramente não há uma tendência da imprensa portuguesa para "preparar; chegar antes de; dispor de maneira que evite (dano, mal); impedir que se realize". Conforme se viu atrás, uma ação preventiva deveria evitar o surgimento de doenças específicas, reduzindo a sua incidência e prevalência, coisa que não acontece no corpus de análise em causa.

Em suma, a participação dos media na promoção da saúde é quase nula e em algumas circunstâncias conseguem até ser contra "certos pressupostos deste processo".

\section{Referências bibliográficas}

ARAÚJO, I. Aprendem doença, educam para a saúde. Universidade do Minho, 2004.

BYDLOWSKI, C.; WESTPHAL, M.; PEREIRA, I. Promoção da Saúde. Porque sim e porque ainda não! Saúde e Sociedade. pp. 14-24, 2004. Disponível em:

$<$ http://www.scielo.br/pdf/sausoc/v13n1/03.pdf $>$. Acesso em: janeiro 2012.

CARCEL, C. Paradigma holístico. Revista trajectos e Projectos, 2. pp.31-35, 2000.

CARVAlHO, G.; GONÇALVES, A.; RODRIGUES, V.; ALBUQUERQUE, C. O modelo biomédico e a abordagem de promoção da saúde na prevenção de comportamentos de risco. Universidade do 
Minho, 2008. Disponível em: <http://repositorium.sdum.uminho.pt/bitstream/1822/7640/1/BM

$\% 20 \% 26 \% 20 \mathrm{HP} \% 20$ comp-risco.pdf> Acesso em: janeiro 2012.

CARVALHO, A. \& CARVALHO. G. Educação para a saúde: conceitos, práticas e necessidades de formação: Lusociência, 2006.

CZERESNIA, D. Promoção da Saúde: conceitos, reflexões, tendências. Rio de Janeiro: Ed. Fiocruz. pp.39-53, 2003.

Dicionário Priberam da Língua Portuguesa (2010). Disponível em:

$<$ http://www.priberam.pt/dlpo/dlpo.aspx?pal=Chave> Acesso em: janeiro 2012.

FERREIRA, A. Novo Dicionário da Língua Portuguesa. Rio de Janeiro : Nova Fronteira, 1986.

FILHO, N. Qual o sentido do termo saúde? 2000. Disponível em: <http://dx.doi.org/10.1590/S0102311X2000000200001> Acesso em: janeiro 2012.

GOMIS, L. Teoría del Periodismo: Cómo se Forma el Presente. Barcelona: Paidós, 1991.

HUBLEY, J. Communicating Health. Londres: MacMillan Education Ltd, 1993.

INGRAM, D.\& HENSHALL, P. The News Manual Online. A professional resource for journalists and the media. Australia, 2008. Disponível em: <http://www.thenewsmanual.net/> Acesso em: maio 2012.

JUNIOR, W. Classificação das bases conceituais para elaboração de sistema digital de busca de fontes jornalísticas. Revista Galáxia, n. 12. pp. 115-128. São Paulo, 2006.

KREPS, G. The impact of communication on cancer risk, incidence, morbidity, mortality, and quality of life. Health Communication, 15. pp. 163-171, 2003.

KREPS, G. \& THORNTON, B. Health communication. New York, NY, Longman Inc, 1984.

KREPS, G. \& NEUHAUSER, L. Rethinking communication in the E-health Era. Journal of Health Psychology. SAGE, 2003.

LEFÉVRE, A. Mitologia sanitária: saúde, doença, mídia e linguagem. São Paulo: Ed. Universidade de São Paulo, 2009.

LOPES, F.; RUÃO, T.; MARINHO, S. Revista Comunicação e Sociedade - Edição especial sobre Comunicação e Saúde. Centro de Estudos em Comunicação e Sociedade, 2012.

LOPES, F.; RUÃO, T.; MARINHO, S.; ARAÚJO, R. Jornalismo de saúde e fontes de informação, uma análise dos jornais portugueses entre 2008 e 2010. Derecho a Comunicar, $\mathrm{n}^{\mathrm{o}}$ 2, pp. $100-120$, 2011.

MARTINS, M. A Promoção da saúde: percursos e paradigma. Instituto Politécnico de Castelo Branco, 2005.

MOISÉS, M. A Educação em Saúde, a Comunicação em Saúde e a Mobilização Social na Vigilância e Monitoramento da Qualidade da Água para Consumo Humano. Jornal do Movimento Popular de Saúde/MOPS, 2003.

MOREIRA, P. Para uma prevenção que previna. Coimbra: Quarteto, 2005.

NORTHOUSE, P. \& NORTHOUSE, L. Health communication: a handbook for health professionals. New Jersey, Prentice Hall, 1985.

NUTBEAM, D. Health literacy as a public health goal: a challenge for contemporary health education and communication strategies into the 21 st century. Health promotion international. Vol. 15. Great Britain: Oxford University Press, 2006.

OMS (Organização Mundial da Saúde). Carta de Ottawa. In: Promoção da Sáude e Saúde Pública ·pp. 158-162, Rio de Janeiro: ENSP, 1986.

PACHECO, J. Comunicação em Saúde: uma necessidade atual, 2005. Disponível em:http://www.comunicasaude.com.br/revista/02/artigos/artigo6.asp Acesso em: janeiro 2012.

PELLEGRINI, P. A atuação das fontes na construção do discurso jornalístico. Revista Cambiassu, $\mathrm{n}$. 4. pp. 269-288, 2008.

RENAUD, L \& SOTELO, C. Comunicación y Salud: Paradgmas Convergentes. Observatorio Journal, 2. pp. 215-226, 2007.

SAINERO, G.; IRURETA-GOYENA, P.; LOPEZ, E. Manuel de fuentes de información. Madrid: CEGAL, 1994.

TEIXEIRA, J. Comunicação em saúde. Relação Técnicos de Saúde - Utentes. Lisboa, 2004. 
TERRÓN, J. El estado de la comunicación y la salud en España, s/d. Disponível em: <http://www.aeic.org/santiago2008/contents/pdf/comunicaciones/245.pdf $>$ Acesso em: janeiro 2012.

THOMAS, R. Health Communication. EUA: Springer Science + Business Media, Inc, 2006.

TILFORD, K. \& TONES, S. Health education. Efectiveness, efficiency and equity. Londres: Chapman \& Hall, 1994

TUFTE, T. La comunicación y la salud en un contexto globalizado. Situación, logros y retos. Encuentro de FISEC, V, 2007. Disponível em:

$<$ http://www.cienciared.com.ar/ra/usr/9/487/fisec7_m2pp39_53.pdf > Acesso em: janeiro 2012.

XAVIER, C. Mídia e saúde, saúde na mídia. In: SANTOS (Ed.), Caderno mídia e saúde pública. Belo Horizonte, 2006 
${ }^{\mathrm{i}}$ Teoricamente, Comunicação em Saúde corresponde a todas as formas de comunicação que têm lugar no sector da saúde, enquanto Comunicação para a Saúde se refere às campanhas de comunicação que visam promover a saúde das populações.

ii Educação em Saúde é um termo que abrange todo o tipo de educação, enquanto a Educação para a Saúde implica uma ideia de educação como meio de prevenção em saúde.

${ }^{i i i} \mathrm{O}$ termo fonte surge do latim fons, fontis, que significa nascente, fonte, água, origem. Da mitologia romana, Fontus ou Fons era uma divindade associada às nascentes e tido como filho do deus Jano (ou Janus) (Dicionário Priberam da Língua Portuguesa, 2010).

iv O Público pertence ao grupo Sonae e é possível caracterizá-lo recorrendo ao seu livro de estilo. "O Público não pretende ter o dom da infalibilidade, mas reclama dos seus jornalistas o menor número de erros e imprecisões" é um dos lemas defendidos. Além disso, "a credibilidade do jornal jogar-se-á tanto na qualidade da sua informação como no saber corrigir as suas próprias falhas — pronta e adequadamente". "O direito de resposta e uma relação transparente com os demais órgãos de comunicação social são igualmente normas de um jornal sério e credível".

${ }^{\vee} \mathrm{O} J N$ é um diário popular português, com sede no Porto e que se encontra sobre a alçada do grupo Controlinveste. O diário completa este ano 123 anos de existência, sendo a região Norte a sua grande área de influência. Defende a "solidez", a "confiança" e a "proximidade com os leitores" como os seus pontos fortes.

vi O Expresso - pertencente ao grupo Impresa - caracteriza-se à luz do seu estatuto editorial. "A liberdade de expressão e a liberdade de informar" são a razão de ser apresentada pelo jornal. Além disso, admite repudiar "qualquer forma de censura ou pressão, seja ela legislativa, administrativa, política, económica ou cultural".

vii Tornou-se necessária a criação de uma nomenclatura de fontes, mais uma vez baseada no projeto onde se insere este estudo. Assim, temos uma classificação em função da Geografia; do Género; da Identificação; do Estatuto: Dentro do Campo da Saúde e Fora do Campo da Saúde; e da Especialidade.

Este artigo e todo o conteúdo da Estudos em Jornalismo e Mídia estão disponíveis em http://www.periodicos.ufsc.br/index.php/jornalismo/index

Estudos em Jornalismo e Mídia está sob a Licença Creative Commons. 